\title{
3D CFD numerical analysis of vane dynamic effects on the pressure ripple in a variable displacement vane pump
}

\author{
Gianluca Marinaro ${ }^{1, *}$, Emma Frosina ${ }^{2}$, Adolfo Senatore ${ }^{1}$, Kim A. Stelson ${ }^{3}$, and Yuhao Feng ${ }^{3}$ \\ ${ }^{1}$ University of Naples “Federico II”, Department of Industrial Engineering, via Claudio 21, 80125 \\ Naples, Italy \\ ${ }^{2}$ University of Sannio, Department of Engineering, Piazza Roma 21, 82100 Benevento, Italy \\ ${ }^{3}$ University of Minnesota, Department of Mechanical Engineering, 111 Church Street SE, 55455 - \\ Minneapolis, MN, USA
}

\begin{abstract}
A numerical three-dimensional CFD analysis of a variable displacement vane pump has been conducted, investigating the effects on the pressure ripple caused by the vane detaching from the pressure ring. The volume of the fluid over the vane tip has been re-meshed at every time step as a function of the forces acting on the bottom and the top of each vane. The numerical model has been developed using the commercial tool, Simerics MP+, including turbulence and cavitation models. The validation of the model has been done comparing numerical and experimental data. It has been observed that the detachment of the vane occurs during the transition zones when unwanted pressure spikes are generated by a nonoptimized valve plate design. The prediction of vane detachment is crucial for designing a quieter and more durable pump. Vane collision on the stator ring can be a source of noise producing premature wear of both components. Vane detachment from the stator ring has a large effect on pressure ripple even if the volumetric efficiency is only slightly influenced.
\end{abstract}

\section{Introduction}

Variable displacement vane pumps are widely used in industrial applications. Vane pumps have desirable characteristics including variable sealing between the vanes and stator ring that can self-compensate for wear through additional vane extension without losing volumetric efficiency. If wear of the vanes tip become excessive, the vanes can easily be replaced. Variable displacement vane pumps are very compact, especially axially and they have very low flow pulsation [1]. To better understand and optimize the fluid dynamics, numerical models can be beneficial to reduce the number of prototype iterations. As detailed below, several numerical studies are available for the understanding the operation of vane pumps in a wide variety of applications.

\footnotetext{
* Corresponding author: gianluca.marinaro@unina.it
} 
Geist et al. [2] analyzed a vane pump used for lubrication of an internal combustion engine. An accurate numerical model has been built to account for the effect of eccentricity rate of change on the internal forces and torques acting on the variable displacement mechanism within a pivoting vane pump. Sullivan et al. [3] focused attention on the internal forces of a variable displacement vane pump. A Computational Fluid Dynamics (CFD) numerical model has been developed showing that pump speed has a greater influence than eccentricity on internal forces, and that the maximum value of the internal moment occurs at high speeds and maximum eccentricity. Other interesting investigations have been done through the years by Rundo et al. [4-5] using both lumped and three dimensional numerical approaches. Rundo developed a customized lumped model implemented in the LMS Amesim ${ }^{\circledR}$ environment that can be used as a predictive tool for evaluating the filling capability, leakage and pressure ripple for a vane pump [4]. In [5], using the same approach, the authors optimized a pump analyzing the effect of the geometry, the position of the silencing grooves, the shape of the vane tip, the clearances, and the precision on the stability of the displacement. With a similar approach, Fornarelli et al. [6] also studied a vane pump using a lumped parameter approach to estimate the effect of friction forces on volumetric efficiency loss, but without supporting experimental tests.

The research presented in this paper is result of a collaboration between three universities that have been working on this topic for many years looking in particular at the prediction of the vane detachment due fluid dynamic effects, and the resulting high-pressure ripple. A three dimensional CFD numerical models has been previously developed by the team [7-8] with good results, but the approach had critical issues that influences construction and calculation times. For this reason, a faster methodology using a lumped approach.

In an unbalanced variable displacement vane pump, an individual displacement chamber is bounded by the rotor and the stator ring in the radial direction and by two consecutive vanes in the circumferential direction. An unbalanced vane pump has an eccentricity between the rotor and stator, which creates the displacement volume. The sliding of the vanes in the rotor slots creates extra displacement volumes and, for this reason, vanes are considered as active elements in the pumping mechanism.

The subject of the study is a prototype vane pump supplied by the Italian company Duplomatic MS; the main technical specifications are listed in Table 1.

Table 1. Technical specifications

\begin{tabular}{|c|c|c|c|}
\hline Geometrical displacement $( \pm 3 \%)$ & $\left(\mathrm{cm}^{3} / \mathrm{rev}\right)$ & 63 & \multirow{7}{*}{ Hydraulic symbol } \\
\hline Theoretical flow rate at $1500 \mathrm{rev} / \mathrm{min}$ & $(\mathrm{L} / \mathrm{min})$ & 94.5 & \\
\hline Maximum continuous pressure & (bar) & 250 & \\
\hline Pressure adjustment range & (bar) & $20 \div 250$ & \\
\hline Rotation speed range & (rev/min) & $800 \div 1500$ & \\
\hline Rotation type & $(-)$ & CCW & \\
\hline Number of vanes $(\mathrm{z})$ & $(-)$ & 11 & \\
\hline
\end{tabular}

The pump is a variable displacement vane pump with a hydraulic operated pressure compensator, which permits instantaneous adjustment of the flow rate to match the application requirements. The pressure compensator operates to keep the stator ring in the required eccentric position actuated by a piston hydraulically controlled by a pressure pilot 
stage. When the delivery pressure equals the pressure corresponding to the pilot stage setting, the stator ring is moved toward the center, adjusting the flow rate to the system requirements. At zero flow demand conditions, the pump delivers oil only to compensate for leaking and piloting, keeping the circuit pressure constant.

The geometrical displacement presented in Table 1 is slightly variable for any particular pump because of production and assembly variations. This type of positive displacement pump is frequently used for industrial application at medium or high-pressure levels. The clearance around the stator ring depends on manufacturing tolerances. To remove this clearance, a screw alters the thrust block position, an offset perpendicular to the pump eccentricity (later called Y-offset). This procedure is performed during pump calibration and breaking-in assuring a working condition with no clearances. The Y-offset can be positive or negative, affecting the maximum and minimum volume angular position of the displacement between vanes, shifting the valve plate timing [4 - 5]. This can cause an unwanted premature closing of the delivery kidney reducing the effective pump displacement below the nominal geometrical displacement from the manufacturer. To create a more accurate numerical model, the authors measured the Y-offset before the pump tests, and considered it in the numerical model.

\section{Experimental setup}

Experimental tests that have been performed on a test rig at the Mechanical Engineering Department of the University of Minnesota. The schematic and the test rig are shown in Fig. 1. A three phase, 150HP motor (1), is used to drive the hydraulic pump (2) test, has been connected to a variable-frequency drive (VFD) (8). The motor has been connected to the hydraulic pump through a torque transducer (Lebow 1225) with its speed controlled by the VFD model ABB ACH550-UH-180A-4. The load valve (3) is an Eaton CMA200 that is an advanced CAN-Enabled electrohydraulic sectional mobile valve with independent metering that utilizes pressure and position sensors, on board electronics, and advanced software control algorithms. The test rig is equipped with a water-oil heat exchanger (6), a filter (5), a tank (7) and a manifold (4) housing a turbine type flow meter, a thermocouple and a fast response pressure transducer. The oil used is ISO VG46. The fluid temperature is controlled to $313 \mathrm{~K}$.

a)

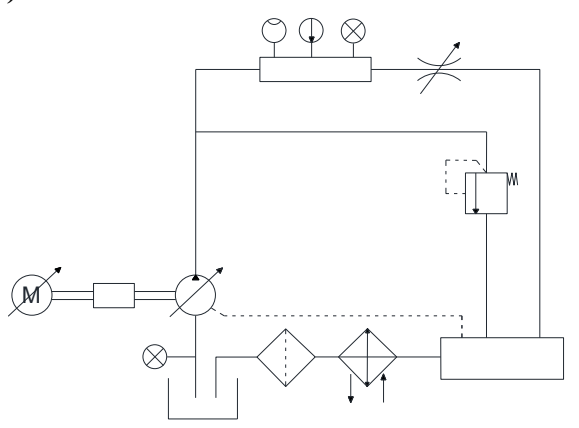

b)

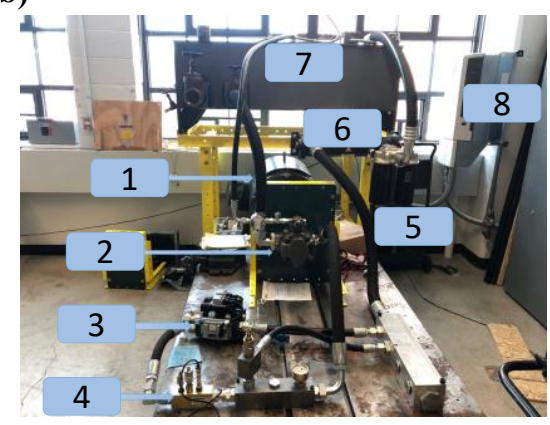

Fig. 1 - Test rig of the University of Minnesota;

a) Schematic of the test stand, b) Set of the hydraulic pump test stand 


\section{Numerical}

The pump has been modeled with the commercial code Simerics MP $+{ }^{\circledR}$, which integrates a rotor template mesher dedicated for vane pumps. The authors integrated a script, that remeshes the vane tip volume every time-step to simulate vane detachment from the stator ring, explained later. The commercial software discretizes the governing equations including conservation of mass and momentum, using a finite volume method. The fluid volume domain has been extracted from the CAD 3D drawing, provided by the company in a STEP format, with the commercial software Discovery SpaceClaim ${ }^{\circledR}$, and divided in sub-volumes, which will be interfaced during modeling in Simerics MP $+{ }^{\circledR}$. To help the interfacing process, surfaces have been renamed in Discovery SpaceClaim ${ }^{\circledR}$ to be easily distinguished. Finally, the fluid domains have been exported in STL format and imported in the CFD code and then meshed with opportune grid sizes. The numerical model does not include heat transfer, the temperature is constant and affects oil viscosity and density; the dynamic viscosity and liquid bulk modulus are, respectively, exponentially and linearly pressure dependent. In industrial fields the flows are almost always turbulent therefore, ideally, Direct Numerical Simulation (DNS) should be adopted to capture all the scales, but the computational time at this Reynolds numbers is very lengthy. Alternatively, other models can be used for the computation of turbulent flow; Large Eddy Simulation (LES) resolves the large eddies, modeling only the small-scale eddies, but it requires a very fine mesh and very small time-step, making it not applicable to industrial application. The faster and very common way is to model turbulence is to use the Reynolds-Averaged Numerical Simulation (RANS), which has been proved to be capable of predicting the correct time-averaged flow field, in particular for wall bounded flows. Simerics MP+ adopts the RANS approach to model the turbulent flow, using the standard "K-Epsilon" and the RNG "K-Epsilon" turbulence models available in the software. The authors selected the standard "K-Epsilon" model, since it previously provided an accurate evaluations of the flow field in applications similar to the pump analyzed in this paper [7-8].

Cavitation is needed for the vane pump model. Since pump timing is fairly variable, conditions can create circumstances where the pressure causes the dissolved air in the liquid can come out of the solution. Since the local pressure affects the vane balance, a cavitation model is needed. Otherwise, if the pressure is negative, the pressure force evaluation on the vane will be inaccurate. The model used for cavitation is based on the advanced cavitation model proposed by Singhal et al. [9], where non-condensable gas (NCG) in both dissolved and undissolved states are considered. In this analysis, the "equilibrium dissolved gas model", integrated in Simerics MP $+{ }^{\circledR}$ and already used by author [6], has been used for the purpose. Here the sum of the dissolved and undissolved mass fraction is constant. A mesh sensitivity analysis has been conducted to obtain the outlet volumetric flowrate independence of cell size, as visible in Fig. 2 this condition has been achieved with a model of around 1M cells, in particular the rotor mesher for the structured vane volumes has been set as follows: 440 cells in the circumferential direction, 8 in the radial direction, 46 in the axial direction, and $5 \times 10$ in the tip gap. A model with finer mesh has been investigated, but the results did not justify the increment in the computational time. 


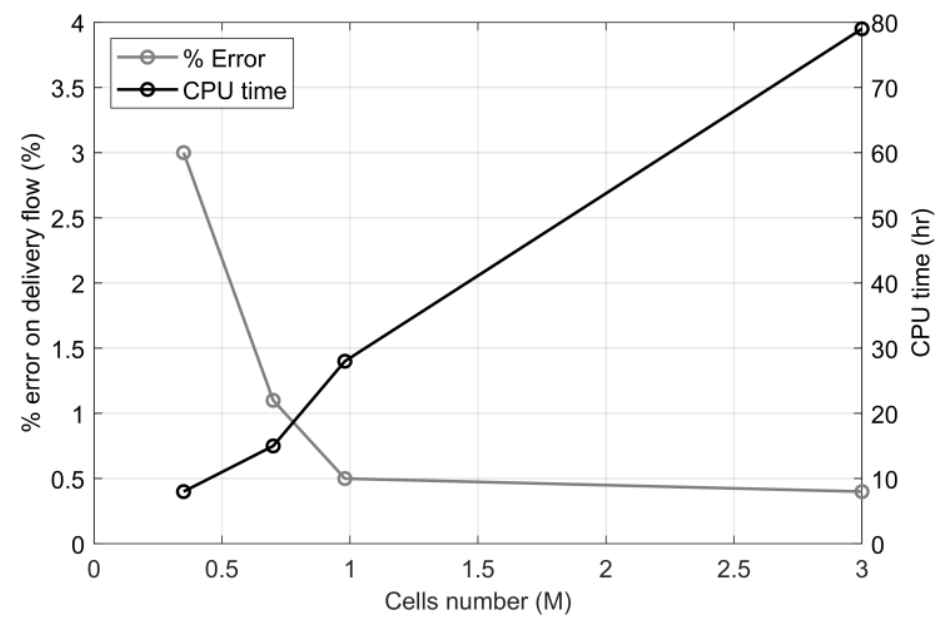

Fig. 2. Mesh sensitivity analysis

An opportune convergence criterion has been set to assure mass flow conservation between inlet and outlet. The number of time step, $\Delta t$, per rotation is 880 , corresponding to an angular step of $0.4 \mathrm{deg}$. The conditions for the simulation are the following:

- Inlet and case pressure: 1.01325 bar abs

- Outlet pressure: $21.01325 \div 251.01325$ bar abs

- Oil type: commercial ISO VG46;

- $\quad$ Temperature: $313 \mathrm{~K}$;

The two axial gaps, between rotating elements and valve plates, have been considered function of the outlet pressure to simulate the "clamping" force exerted by the two valve plates due to the pressure balance on their two surfaces. This is designed to maintain a high volumetric efficiency at higher pressures. The script introduced by the authors, that models the vane detachment from the stator ring, works following eq.(1). The possibility of detachment is evaluated by a force balance between two terms, one includes the effect of the pressure force coming from the under-vane added to the centrifugal force while the other evaluates the radial pressure force acting on each vane. The forces are shown in Fig. 3.

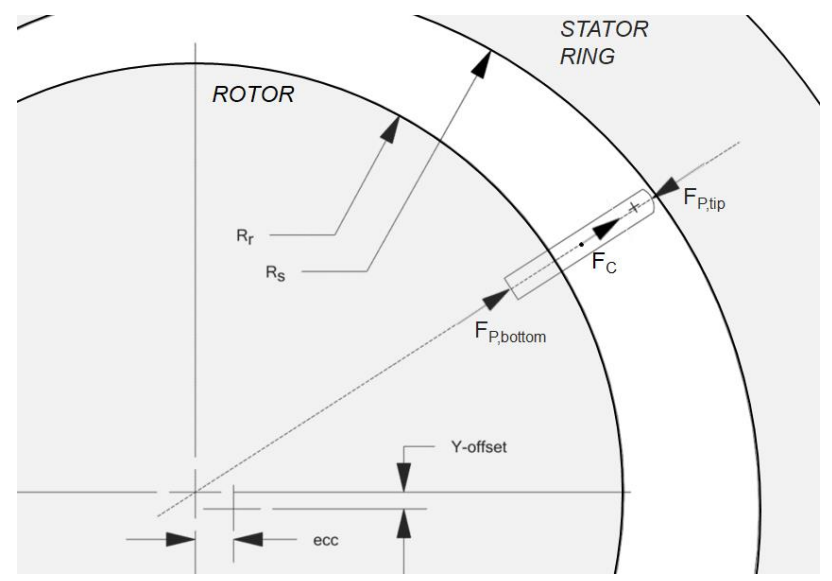

Fig. 3 - Force considered for the vane balance 


$$
F_{\text {contact }}(t)=\left(F_{p, \text { bottom }}(t)+F_{C}(t)\right)-F_{p, \text { tip }}(t)
$$

Where $F_{\text {contact }}$ is the contact force between the vane and the stator ring, $F_{p, \text { bottom }}$ and $F_{p, t i p}$ are the pressure forces acting on the bottom and top of the vane, $F_{C}$ is the centrifugal force on the vane; friction forces are neglected. If the value $F_{\text {contact }}$ at time $t$ is not negative, the tip gap remains constant and equal to the minimum value at the time $(t+\Delta t)$, otherwise the script remesh the vane tip gap $h_{\text {tip }}$ according to a scaling factor, $\alpha$.

$$
\left\{\begin{array}{cc}
h_{\text {tip }}(t+\Delta t)=h_{\text {tip }, M I N}, & F_{\text {contact }}(t) \geq 0 \\
h_{\text {tip }}(t+\Delta t)=\alpha\left(F_{\text {contact }}(t)\right) \cdot h_{\text {tip,MIN }}, & F_{\text {contact }}(t)<0
\end{array}\right.
$$

In eq. (2), the scaling factor has been tuned to reach a smooth detachment avoiding an unrealistic gradient on the vane displacement. The scaling factor is a function of the ratio between the maximum $F_{\text {contact }}$ and the maximum allowable vane displacement. This calculation has been done for each of the eleven vanes.

In Fig. 4a) the mesh of the pump fluid domain is visible. In the zoomed view the reference under-vane and main chamber volumes at starting angle ( 0 rad) have been shown. This will be helpful for understanding of the polar plots presented later. The nomenclature for the two vanes is also shown. Fig. 4b) shows an exaggerated view of how the script integrated by the author works to simulate the vane detachment.

a)

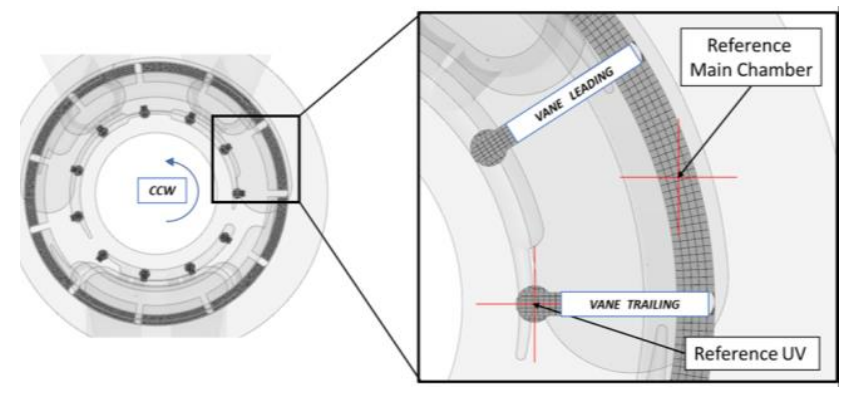

b)

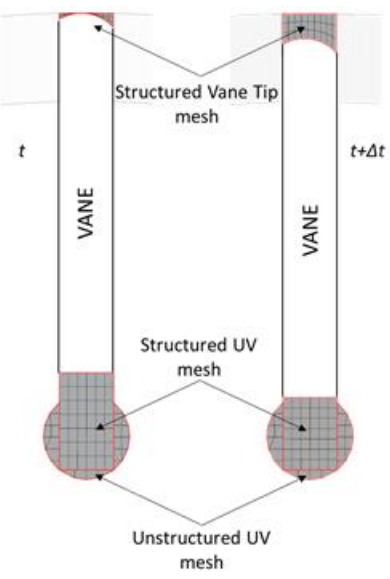

Fig. 4. a) Mesh of the fluid domain at zero displacement with nomenclatures in the zoomed view b) Example of the vane re-mesh from time $t$ to time $t+\Delta t$ if the contact force is negative.

With reference to Fig. 3b, two volumes at the vane top and bottom can be seen. Both volumes have been modeled with a structured mesh. This is very helpful for remeshing, when cells need to be stretched along an orthogonal direction. If the quantity evaluated by equation (1) becomes negative, the two volumes are re-meshed to simulate vane detachment from the stator ring. The structured mesh of the under-vane (UV) is re-meshed every time step to follow the eccentricity of the stator ring. 


\section{Model Validation}

Once the mesh sensitivity analysis has been completed, the model can be validated on experimental data. Simulation have been conducted on a desktop workstation equipped with an Intel ${ }^{\circledR}$ Xeon ${ }^{\circledR}$ CPU E5-2640 v2, 16 cores; where each run took around seven hours for a pump revolution. In Fig. 5 experimental and numerical results have been presented on a flowpressure diagram; three rotational speeds have been analyzed (1000, 1200, $1500 \mathrm{rev} / \mathrm{min})$.

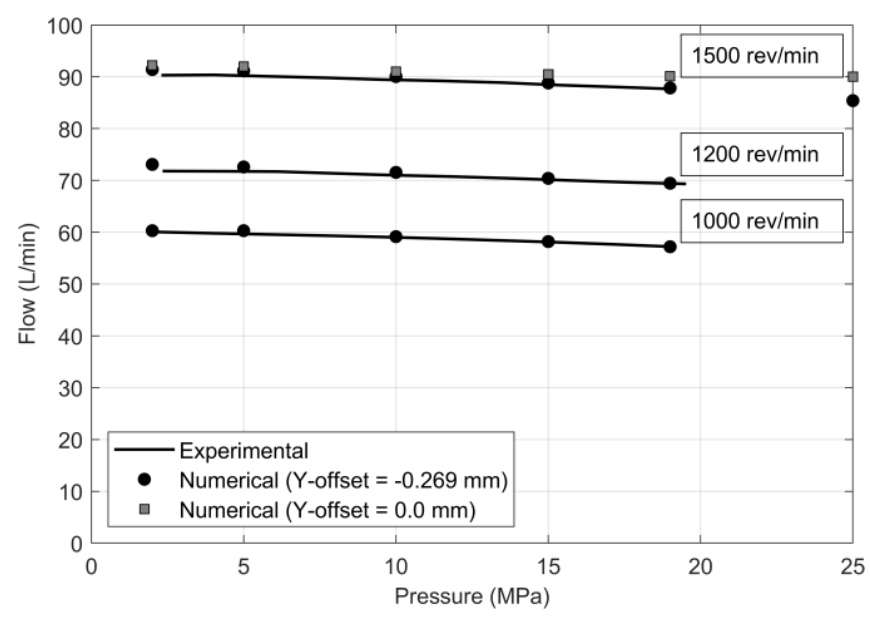

Fig. 5. Numerical model validation

The need to consider the Y-offset is clearly visible for the $1500 \mathrm{rev} / \mathrm{min}$ curve. Acting on the valve plate timing, the offset reduces the effective pump displacement. Considering the measured Y-offset and the tuned scaling factor, useful for the vane detachment re-meshing, the errors are within $1 \%$ of the experimental data for all rotational speeds. Fig. 5 clearly shows the importance of considering the Y-offset. St the same time it is important to visualize the effects of the vane detachment on the pressure ripple, which is the main purpose of this study. Experimental data have been acquired using a calibrated orifice [10-11]. This methodology creates a system load, that can be easily implemented in the numerical model.
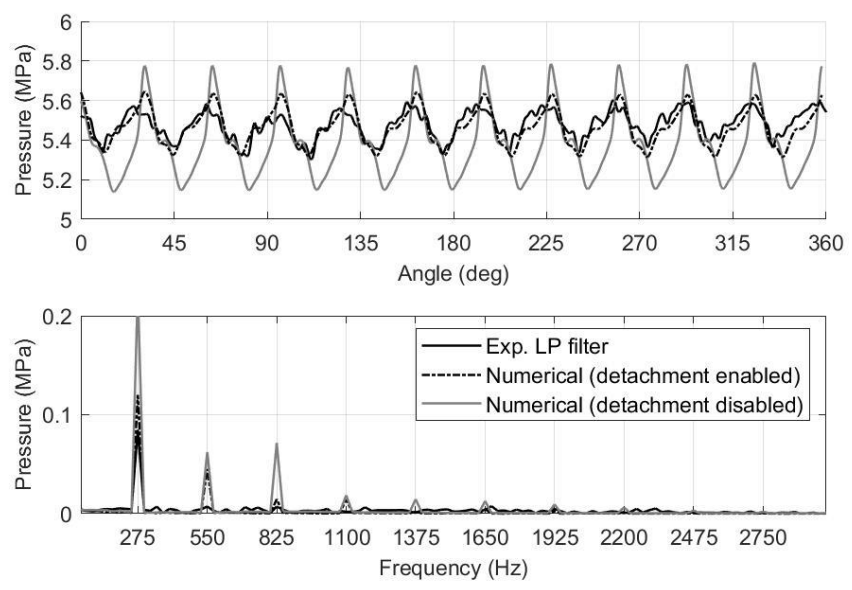

Fig. 6. Pressure ripple 
In Fig. 6 experimental pressure ripple acquired at $50 \mathrm{kHz}$ - filtered with a 70th order lowpass FIR with a cutoff frequency of $3 \mathrm{kHz}$ - and numerical pressure ripples, with and without the vane detachment script, have been presented in both time (angle) and frequency domains.

Fig. 6 also reveals the importance of considering vane detachment for an accurate analysis. The amplitude of the pressure ripple oscillation (visible on the 1st fundamental frequency) is quite different from experimental data if the script is not enabled. Vane detachment has to be considered if the pressure/flow ripple is analyzed. Otherwise an overestimation will result.

\section{Results}

Polar plots have been created by the authors to present numerical simulation results. The valve plate shadow has been added as background for better understanding. The internal smaller kidneys are related to the under-vane timing, while the external bigger ones to the main chamber; for all the following figures the upper side is connected to the pump outlet, while the lower side is linked to the pump inlet.

In Fig. 7 the displacement volumes have been plotted as function of angle. As expected, the under-vane maximum and minimum volumes occurs at 0 and $\pi$ radians, while the maximum volume of the main chamber is not aligned to $-\pi / z$ because it is shifted by a value of around $\pi / 36$ ( $5 \mathrm{deg}$ ) caused by a not-null Y-offset.

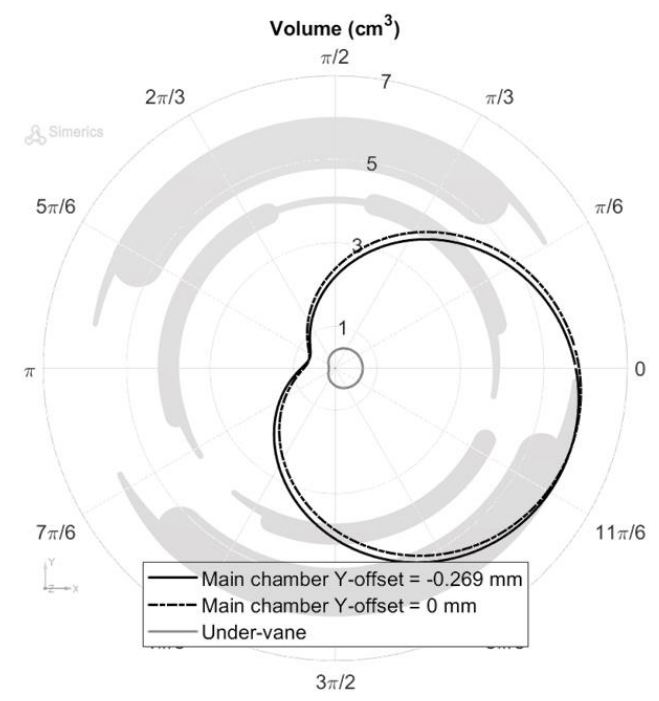

Fig. 7. Displacement chambers volume polar plot

In Fig. 6, both main chamber volumes, with and without the Y-offset have been shown. Since this dimension highly influences the pump fluid dynamics, all the following results considers the measured value of the Y-offset of $0.269 \mathrm{~mm}$. 
a)

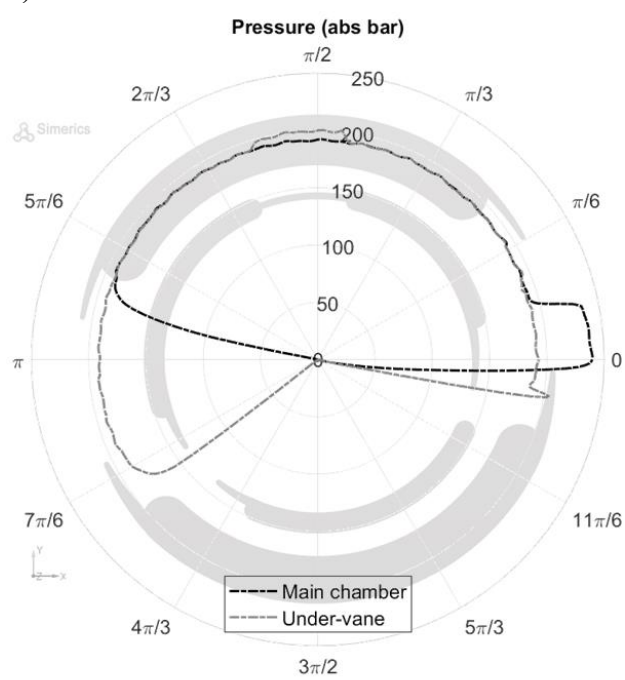

b)

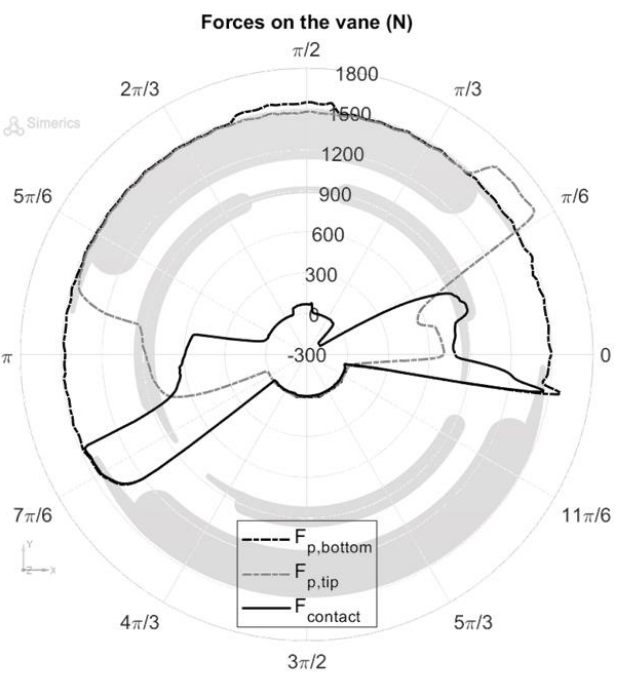

Fig. 8. a) Displacement volumes pressure - b) Forces on the vane polar plots

In Fig. 8a) the pressure trends of the main chamber and under-vane volumes have been presented. It is clearly visible how the under-vane chamber pressure builds up before the initial angle ( $0 \mathrm{rad})$ and it is still in pressure after $\pi \mathrm{rad}$ to create a proper sealing between the pump inlet and outlet. In Fig. 8b) $F_{\text {contact }}$ is shown. It achieves a maximum value of around $1500 \mathrm{~N}$ while becomes negative in the angular region between $\pi / 6$ and $\pi / 3$. The pressure spike in the reference main chamber creates a detachment of the leading vane (Fig. 4a).

a)

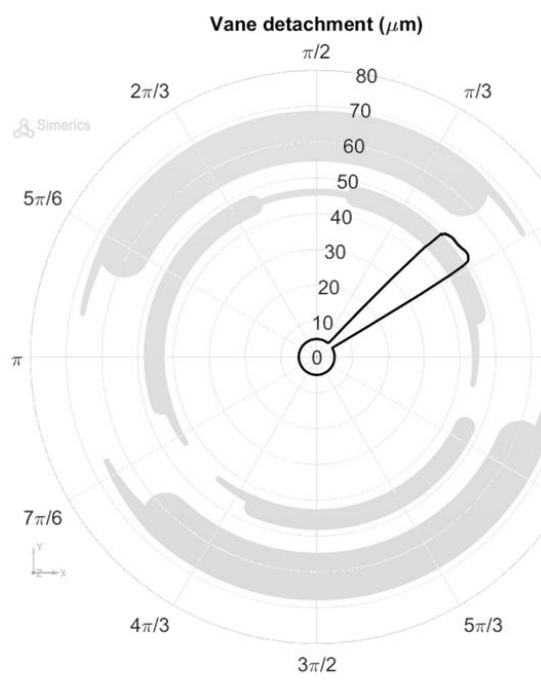

b)

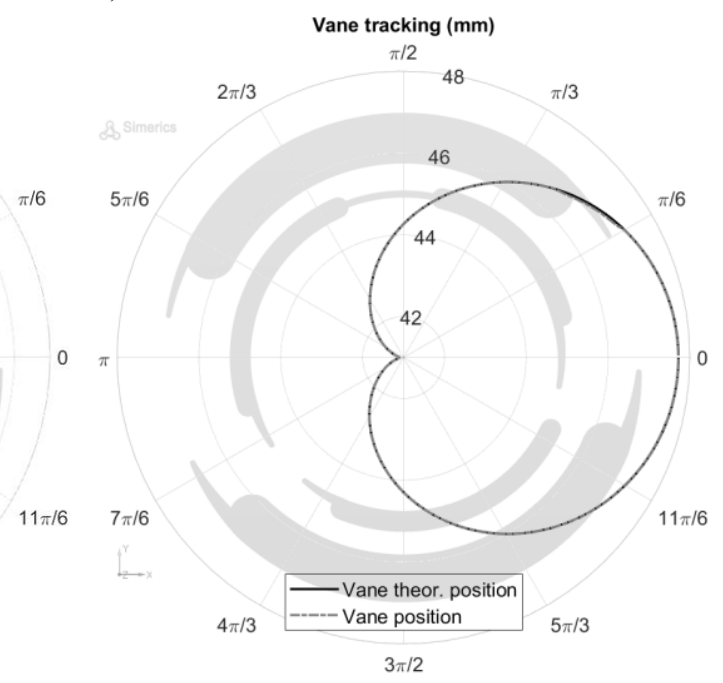

Fig. 9. a) Detachment - b) Tracking for the trailing vane. 
The Fig. 9a show the calculated vane detachment from the stator ring, the estimate is around $50 \mu \mathrm{m}$ occurring in the same angular region where $F_{\text {contact }}$ becomes negative. In Fig. $9 \mathrm{~b}$, tracking of the instantaneous and theoretical vane position is visible, including with or without the vane detachment script.

Another interesting result has been shown in the Fig. 10, where the stator ring is presented in three views. The pictures have been taken during a preliminary disassembly, coincident with the acquisition of the Y-offset, so at that time the pump only did the running in, for this motivation the signs can be more accentuated after some hour in working condition. In the middle picture of Fig. 10, the contact force polar plot has been inserted. Looking at the same colors' squares, signs of the contact between vane and stator ring can be noted.
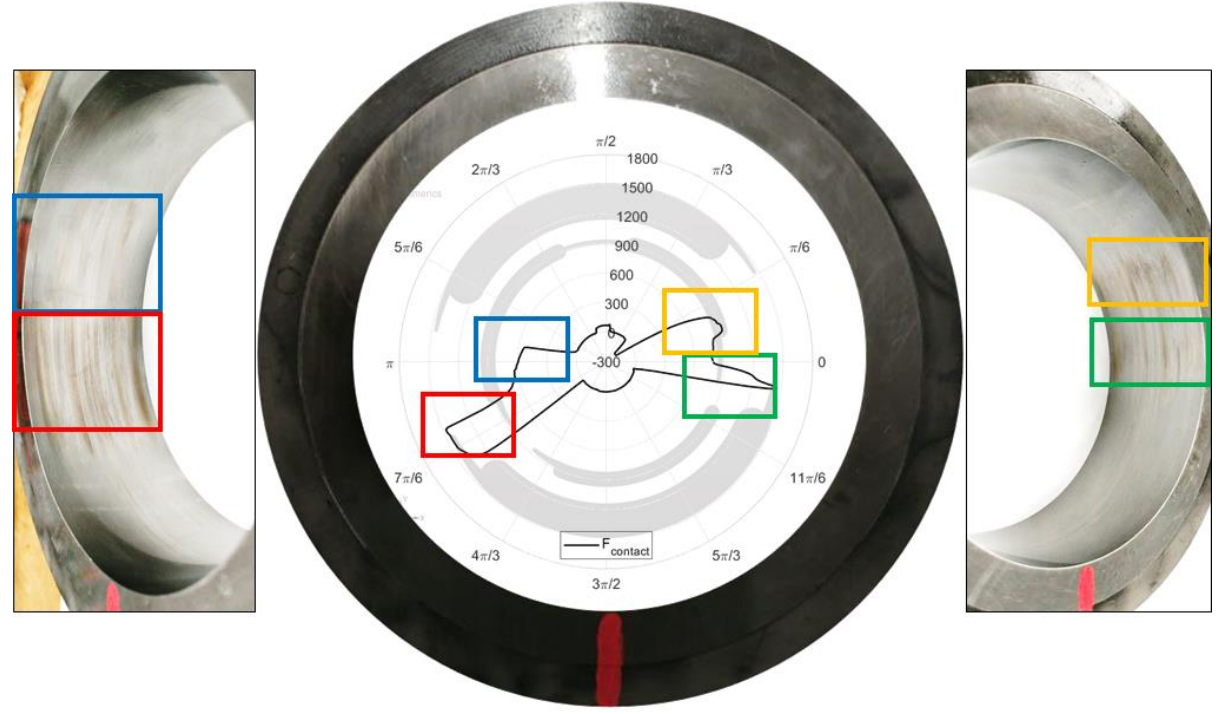

Fig. 10. Stator ring projections with integrated plot for contact force.

The left view picture identifies the transition region from delivery to suction, in the blue square, the area of the ring where there is a partial contact force (around 600N) has been evidenced; scratches are evident in this area. In the same view, the area identified by the red square presents important signs, more than the previous blue area, here the contact force achieves maximum values (almost 1500N). On the other side, during suction to delivery transition, same signs are evident in the green highlighted region, where for a moment the contact force achieves the maximum value. In the yellow area, just before the vane detachment, it is clearly visible a densification in a well identified angular strip, here the force increases to a value of $900 \mathrm{~N}$. This qualitative observation further confirms the predictive ability of the methodology.

\section{Conclusion}

A study on an unbalanced variable displacement vane pump for industrial application has been presented. The pump has been supplied by the Italian company Duplomatic MS and tested at the University of Minnesota. Numerical analysis has been conducted in a threedimensional CFD environment with the commercial software Simerics $\mathrm{MP}+{ }^{\circledR}$ at the 
University of Naples "Federico II" and the University of Sannio. Effects of vane detachment and Y-offset presence have been investigated. The mesh built by the rotor template mesher, included in the commercial code, has been modified at every time step with an intergrated script created by the authors.

The presented results confirmed the accuracy of the methodology, which can be used during the design process to control the contact force to increase component lifetimes. An overestimation on the pressure ripple has been found if detachment is ignored in the pump. For the analyzed design, no problems of vane collision on the stator ring have been found. Detachment has been observed in the numerical model, after that the contact force became very small. The Y-offset has to be considered during valve plate timing design, since this value can be variable because of component dimensional variations. This timing change can dangerously change pump behavior. The Y-offset lies in a certain range, a function of exact dimensions for an individual pump. For this reason, the pump timing has to be designed to compensate for production dimensional variations on final parts. Methodologies, like the one presented in this study, can be very helpful during the design process, being cost effective and reducing prototyping time.

\section{Acknowledgements}

We appreciate the support from Michele Pavanetto and Alessandro Corrente of Duplomatic MS, from Raffaele Invrea and Travis Piehl of Continental Hydraulics Inc. and from Federico Monterosso and Micaela Olivetti of OMIQ s.r.l.

\section{Nomenclature}

\begin{tabular}{ll}
$F_{C}$ & Vane centrifugal force \\
$F_{\text {contact }}$ & Contact force between vane and stator ring \\
$F_{p, \text { bottom }}$ & Pressure force acting on vane bottom radially \\
$F_{p, t i p}$ & Pressure force acting on vane tip radially \\
$h_{\text {tip }}$ & Vane tip gap dimension \\
$h_{\text {tip,MIN }}$ & Vane tip gap minimum dimension \\
\multicolumn{1}{c}{ Greek letters } & \\
$\alpha$ & Scaling factor \\
\multicolumn{1}{c}{ Abbreviations } & \\
CAD & \\
CCW & Computer-aided design \\
$C F D$ & Counterclockwise \\
$F I R$ & Computational fluid dynamics \\
$S T E P$ & Finite impulse response \\
$S T L$ & Standard for the Exchange of Product Data \\
UV & Standard Triangle Language
\end{tabular}




\section{References}

1. J. Ivantysyn, M. Ivantysynova, Hydrostatic Pumps and Motors: Principles, Design, Performance, Modelling, Analysis, Control and Testing. Tech Books International (2003).

2. B. Geist, W. Resh, Dynamic Modeling of a Variable Displacement Vane Pump Within an Engine Oil Circuit, Proceedings of the ASME 2011 Internal Combustion Engine Division Fall Technical Conference ICEF2011 October 2-5, 2011, Morgantown, West Virginia, USA.

3. P. E. Sullivan, M. Sehmby, Internal Force Analysis of a Variable Displacement Vane Pump, SAE Technical Paper 2012-01-0409, 2012.

4. M. Rundo, M. A. Pavanetto, Comprehensive Simulation Model of a High Pressure Variable Displacement Vane Pump for Industrial Applications, Proceedings of the ASME 2018 International Design Engineering Technical Conferences and Computers and Information in Engineering Conference IDETC/CIE 2018, August 26-29, 2018, Quebec City, Quebec, Canada.

5. M. Rundo, G. Altare, Lumped Parameter and Three-Dimensional CFD Simulation of a Variable Displacement Vane Pump for Engine Lubrication, Proceedings of the ASME 2017 Fluids Engineering Division Summer Meeting FEDSM2017 July 30-August 3, 2017, Waikoloa, Hawaii, USA.

6. F. Fornarelli, A. Lippolis, P. Oresta, A. Posa, Investigation of a pressure compensated vane pump, Proceeding of the $73^{\text {rd }}$ Conference of the Italian Thermal Machines Engineering Association (ATI 2018), 12-14 September 2018, Pisa, Italy.

7. E. Frosina, A. Senatore, D. Buono, K. A. Stelson, F. Wang, B. Mohanty, M. J. Gust, Vane pump power split transmission: three dimensional computational fluid dynamic modeling, Proceedings of the ASME/BATH 2015 Symposium on Fluid Power and Motion Control, FPMC2015, Chicago, Illinois, USA.

8. E. Frosina, A. Senatore, D. Buono, K. A. Stelson, F. Wang, B., Gao, H., A ThreeDimensional CFD Methodology to Study Vane-Ring and Vane-Under-Vane Interactions of a Vane Pump Power Split Transmission, $9^{\text {th }}$ FPNI Ph.D. Symposium on Fluid Power (FPNI2016), Florianópolis, Brazil.

9. A.K. Singhal, M.M. Athavale, H.Y. Li, Y. Jiang, Mathematical basis and validation of the full cavitation model, Transactions of the ASME - Journal of Fluid Engineering, vol. 124, pp. 617-624, 2002.

10. E. Frosina, G. Marinaro, A. Senatore, Experimental and Numerical Analysis of An Axial Piston Pump: A Comparison Between Lumped Parameter And 3D CFD Approaches, Proceedings of the ASME-JSME-KSME 2019, $8^{\text {th }}$ Joint Fluids Engineering Conference AJKFluids2019, July 28-August 1, 2019, San Francisco, CA, USA.

11. E. Frosina, G. Marinaro, A. Senatore, M. Pavanetto, Effects of PCFV and PreCompression Groove on the Flow Ripple Reduction in Axial Piston Pumps, 2018 Global Fluid Power Society PhD Symposium, GFPS 2018. 